\title{
LeGes
}

Fabienne Sastre Duret

\section{Comment assurer la participation de publics vulnérables dans une évaluation?}

Selon le droit international et le droit suisse, la prise en compte de l'opinion des enfants et des jeunes dans les actions publiques qui les concernent est garantie. L'exercice de la participation, dans leur cas, comporte de nombreux défis. Ce compte-rendu présente deux exemples d'évaluation. Il montre les avantages et inconvénients de cette implication, discute des enjeux méthodologiques et éthiques et propose des pistes pour intégrer ce public dans une évaluation. La contribution montre les difficultés rencontrées et les compétences au sein des équipes d'évaluation qui permettent de les surmonter. Enfin, elle questionne le positionnement des équipes face aux attentes suscitées par la participation.

Catégories d'articles : Contributions scientifiques du Congrès de la SEVAL 
[1] La participation en évaluation fait l'objet de controverses. Ceci a été largement illustré par les différentes présentations du Congrès 2020. En ouverture de l'atelier, le modérateur Jérôme Mabillard ${ }^{1}$ souligne que la prise en compte de l'opinion des enfants et des jeunes dans les questions ou procédures les concernant est garantie par le droit international ${ }^{2}$. Ces dispositions ont été reprises dans le droit suisse, dans le Code civil par exemple.

[2] Pourtant, le droit à la participation des enfants n'est pas appliqué de manière satisfaisante en Suisse. Un récent rapport du Conseil fédéral met en évidence des lacunes dans le droit de la famille, de la santé, etc. ${ }^{3}$ Ces constats montrent la nécessité de mieux associer ce public. C'est notamment le cas dans les démarches d'évaluation. Faire participer des enfants et des jeunes dans des recherches ne va toutefois pas de soi.

[3] Dans cet atelier consacré aux publics difficiles à atteindre ou fragiles, deux chercheuses présentent des cas concrets ayant impliqué des enfants et des jeunes. Le présent compte-rendu met en évidence leurs apports, puis la discussion qui a suivi. Il met en lumière certains enjeux méthodologiques et éthiques associés à une telle démarche mais aussi certains obstacles à considérer, ainsi que quelques compétences nécessaires pour les évaluateur-trice-s. Enfin, il aborde la question de la responsabilité particulière de l'équipe d'évaluation dans ce type de démarche.

Le projet pilote de promotion de la lecture « Encore! Des Histoires!» (DUNYA ACKLIN, Professeure associée à la Haute école de travail social de Fribourg) :

[4] Le financement du projet pilote de bibliothèque interculturelle de Fribourg (promotion de la lecture en plein air et prêt de livres dans différentes langues) ${ }^{4}$ était conditionné à la réalisation d'une évaluation externe. Les résultats devaient permettre de se déterminer sur la poursuite du projet et son éventuelle réplicabilité. Dans le cadre de l'évaluation finale (2017), les enfants, principaux destinataires du projet, ont été impliqués. L'équipe d'évaluation a récolté leur avis par rapport à leur motivation et à leur satisfaction. Aux plus grands, elle a demandé leurs attentes et leur appréciation sur les possibilités d'amélioration du projet. L'enjeu consistait à faire participer des enfants allophones âgés jusqu'à 14 ans et qui n'étaient pas forcément scolarisés.

[5] Deux modalités de récolte de données ont été retenues. La première a consisté à élaborer un questionnaire adapté à un public hétérogène d'enfants âgés de 3 à 14 ans, parfois non scolarisé et pouvant être mobilisé dans un environnement non structuré (accueil à bas seuil), dans le cadre d'activités en plein air. Le questionnaire a été rempli par l'équipe d'évaluation sur la base des réponses des enfants à des situations imagées au moyen de "smiley » et de bouteilles différemment remplies pour mesurer la fréquence. La deuxième s'est faite sous la forme d'un focus group réalisé avec une dizaine d'enfants âgés de 10 à 14 ans, scolarisés, capables de s'exprimer en français et d'avoir un recul majeur sur leur participation au projet. Dans les deux groupes, la participation a été illustrée ou formalisée, afin de montrer que leur avis serait transmis aux adultes responsables du projet. Les plus jeunes ont mis leur questionnaire dans une urne symbolique. Les plus âgés ont eu la possibilité de rédiger des messages à l'attention des adultes.

Collaborateur scientifique à la Haute école de travail social de Genève.

https://www.unicef.fr/sites/default/files/convention-des-droits-de-lenfant.pdf, article 12 et RS/CH 0.107, article 12 .

3 https://www.parlament.ch/centers/eparl/curia/2014/20143382/Bericht\%20BR\%20F.pdf.

4 https://www.hets-fr.ch/files/prestations/pdf/RM/RM_29_LIVRECH.pdf. 
[6] L'organisation de la récolte des données a posé des défis sur les plans pratique et éthique comme, par exemple, l'accès à un public volatile (cf. participation volontaire au projet) et l'obtention de l'autorisation des parents dans un contexte de migration (17 langues d'origine). Parmi les atouts pour réussir à nouer des relations de confiance, tant avec les enfants que les personnes impliquées dans le projet et issues de différentes communautés, la chercheuse mentionne des compétences en travail social. Parmi les difficultés rencontrées dans ce contexte avec une approche qualitative, elle relève notamment les contraintes temporelles et budgétaires. Ainsi, le temps nécessaire pour développer un outil adapté aux plus jeunes et établir des relations de confiance a été largement sous-estimé. Il n’a pas été totalement comptabilisé. Par ailleurs, il a fallu privilégier un certain pragmatisme propre à la recherche-action auprès des enfants par contraste avec les exigences de rigueur scientifique d'une recherche fondamentale. Ainsi, l'outil n'a pas pu être testé ni adapté en fonction du développement cognitif lié à l'âge des enfants.

\section{A la recherche de méthodologies favorisant la prise de parole citoyenne (Sylvia Garcia} Delahaye - professeure assistante à la Haute école de travail social de Genève)

[7] La chercheuse évoque tout d'abord le cadre de référence de ses travaux en lien avec l'évaluation des politiques publiques ${ }^{5}$. En particulier, elle mentionne le modèle d'évaluation participative en travail social utilisé à la Haute école de travail social (HETS) de Genève. Ce dernier vise à donner du pouvoir aux participant-e-s au travers d'une dimension participative, transformative et émancipatrice. Il s'agit ainsi de créer les conditions de la construction de la capacité d'action du public selon un modèle en trois actes. Le "pré-acte » consiste à co-construire la problématique (le problème à évaluer), en amont de l'évaluation, avec une variété d'acteur-trice-s. Cela concerne en particulier les objectifs et les indicateurs, ainsi que le choix des outils méthodologiques. Ensuite, la récolte de données (l'« acte ») est réalisée avec les acteur-trice-s. Le post-acte prend la forme d'un bilan participatif et collectif comprenant l'analyse des données, l'évaluation de l'atteinte des objectifs, la décision sur la façon de rendre compte et de valoriser les résultats, ainsi que l'examen critique du processus d'évaluation.

[8] Avec l'exemple d'une analyse des besoins des requérant-e-s d'asile mineur-e-s non accompagné-e-s (RMNA) à Genève ${ }^{6}$, la chercheuse montre l'importance de co-construire les connaissances en intégrant le point de vue des jeunes. Ce mandat faisait suite à une recommandation de la Cour des comptes dans un audit montrant que ces besoins n'avaient jamais été spécifiquement analysés, formalisés, validés ni acceptés par l'ensemble des parties prenantes ${ }^{7}$. L'enjeu était alors de permettre l'expression de la voix d'adolescent-e-s qui, de par leur double statut de mineur-e-s et de réfugié-e-s, n'ont peu ou pas de place dans l'espace social.

[9] L'une des difficultés de départ a été d'arriver à créer des liens de confiance avec des jeunes déjà souvent déçu-e-s par les promesses non tenues des autorités. Il a fallu les convaincre que leur participation n'était pas un alibi. Il a fallu aussi trouver une méthode adaptée à leurs capacités d'expression, en l'occurrence l'outil méthodologique « Ma voix en image » déjà testé avec des

5 Childhood studies, définition de la participation en animation socio-culturelle (par ex. Paolo Freire, Jean-Claude Gillet, ...), etc.

6 https://www.hesge.ch/hets/sites/default/files/actualite/documents/rapport_rmna_final_septembre_2019.pdf.

7 https://asile.ch/wp-content/uploads/2018/02/Rapport-136-Requerants-mineurs-non-accompagnes-RMNA-1. pdf. 
adolescent-e-s sous mesures de protection dans le canton de $\operatorname{Vaud}^{8}$. Cet outil a permis d'illustrer la réponse à la question "Comment te sens-tu accueilli-e à Genève et soutenu-e par les professionnel-le-s qui t'entourent? » sous la forme de dessins et d'une mise en scène photographiée. Les commentaires des autres jeunes sur les photos ont permis de formuler des messages clés à adresser aux autorités. Les photos et messages ont été inclus dans un rapport « Regards croisés » qui intégrait aussi le point de vue des professionnel-le-s ${ }^{9}$. Lors de la restitution des résultats, il a été décidé avec les jeunes de leur donner une place dans l'espace public sous la forme d'une exposition de photos en face du Palais Wilson, siège du Haut-commissariat aux droits de l'homme. C'était ainsi une façon d'amener leur parole devant un lieu emblématique des Nations Unies à l'occasion du $30^{\text {ème }}$ anniversaire de la Convention des droits de l'enfant.

[10] Après le mandat, grâce au soutien financier de la HETS, l'équipe de recherche a encore travaillé avec un groupe de jeunes qui souhaitaient présenter leurs messages lors de la Célébration $\mathrm{du} 30^{\mathrm{ème}}$ anniversaire au Palais des Nations unies. Pour éviter d'être identifié-e-s et ainsi risquer de faire échouer leur demande d'asile, les jeunes ont tenu une vidéoconférence, le visage protégé par des masques, ce qui leur a permis un échange valorisant avec les participant-e-s à la Conférence. Le lendemain, c'est un jeune volontaire qui a porté la voix des RMNA devant la Haute commissaire aux droit humains. Il a ainsi transmis leurs demandes, dont la première était d'être avant tout considéré-e-s comme des enfants avec des besoins spécifiques. Ces approches ont favorisé la restauration de la capacité d'action des RMNA.

\section{Discussion avec les participant-e-s à l'atelier}

[11] La participation en évaluation permet de donner une place à la voix des publics inaudibles sur la scène publique de par le statut qui leur est attribué par le cadre légal ou la société. Il arrive cependant que les recommandations d'une évaluation ne soient pas suivies d'actions concrètes par les pouvoirs publics concernés et cela malgré l'intention annoncée au départ.

[12] La discussion porte alors sur l'aspect éthique du risque de susciter des attentes qui ne seront peut-être pas satisfaites. Cela est notamment ressorti d'évaluations participatives avec différents publics (ex. personnes toxicodépendantes). Plusieurs participant-e-s sont pourtant d'avis que cela peut procurer une forme d'empowerment et de reconnaissance. Les résultats de la recherche peuvent faire changer les choses pour les prochains. L'équipe d'évaluation n'a aucune prise sur ce qui va réellement changer mais, si elle ne porte pas la voix de ces publics, les choses ne vont pas évoluer. On touche ici à la dimension citoyenne de l'évaluation.

[13] Il est cependant évident que ces publics fragiles peuvent se sentir trahis ou lâchés. Dans le cas de l'évaluation sur les besoins des RMNA, l'équipe de recherche issue du domaine du travail social a, par la suite, poursuivi un accompagnement des jeunes en continuant à se rendre disponibles pour eux. Cette disponibilité, une fois le mandat terminé, est toutefois impossible à offrir pour de nombreuses équipes d'évaluation, notamment pour des raisons matérielles.

[14] Dans une démarche d'évaluation qui associe des publics vulnérables, l'équipe de recherche peut se trouver confrontée à la réaction des professionnel-le-s qui estiment tout connaître sur

8 https://www.vd.ch/fileadmin/user_upload/organisation/dfj/spj/fichiers_pdf/Regard_crois\%C3\%A9_participation_-_protection_SPJ_juin_2019FINAL2.pdf.

9 https://www.hesge.ch/hets/sites/default/files/actualite/documents/rapport_rmna_final_septembre_2019.pdf. 
leur situation, se déclarent experts et ne voient pas l'intérêt d'interroger les jeunes. L'outil «Ma voix en image » a précisément été conçu pour contourner un obstacle créé par les professionnelle-s. Cela étant, associer les professionnel-le-s à l'évaluation par une méthodologie qui croise les regards sur une problématique leur permet d'évoluer et d'accepter la légitimité de la position des jeunes. La dimension participative permet également de faire entendre la voix des professionnelle-s d'une manière qui sera mieux reçue si elle bénéficie d'un accompagnement scientifique. Cela contribue aussi à valoriser leur rôle comme acteur-trice-s du changement.

\section{Conclusion}

[15] Les présentations et la discussion qui a suivi ont mis en évidence la nécessité d'associer les publics concernés par une action publique, malgré les difficultés dues à leur situation (ex. statut) ou à de faibles compétences en expression écrite ou orale. Ainsi que l'évoquent les standards de la SEVAL ${ }^{10}$, il est nécessaire de prendre en compte au mieux leurs besoins et leurs intérêts. L'atelier a permis d'identifier de nombreux obstacles tels que l'âge, la connaissance de la langue, le statut légal et le rôle de certains professionnel-le-s au contact avec des publics fragiles qui pensent mieux connaître une situation que ces publics eux-mêmes. Pour surmonter ces obstacles, les équipes d'évaluation ont avantage à pouvoir mobiliser des compétences telles que de la créativité sur le plan méthodologique, des savoirs relationnels, une capacité en accompagnement de publics vulnérables, etc. Favoriser la participation de ces publics plus difficiles à atteindre et obtenir des données pertinentes demande une grande disponibilité mais aussi une capacité à établir un lien de confiance. Ce lien peut être mis à mal si les attentes suscitées ne peuvent être satisfaites du fait que les réponses finalement apportées aux problèmes mis en lumière n'appartiennent pas aux équipes d'évaluation mais aux mandants. En ce sens, les équipes d'évaluation endossent une responsabilité pour laquelle elles devront aussi se positionner.

Fabienne Sastre Duret, conseillère scientifique, Direction générale de la santé (canton de Genève), membre du GREVAL.

10 https://www.seval.ch/app/uploads/2018/01/Standards-SEVAL-2016_f.pdf. 